\title{
YET MORE ON THE DIFFERENTIABILITY OF CONVEX FUNCTIONS
}

\author{
JOHN RAINWATER
}

(Communicated by William J. Davis)

\begin{abstract}
Generic differentiability theorems are obtained for convex functions which are defined and locally Lipschitzian on the convex subset $N(C)$ of nonsupport points of a closed convex subset $C$ of a Banach space $E$, which is assumed to be either an Asplund space (for Fréchet differentiability) or to be weakly compactly generated (for Gateaux differentiability).
\end{abstract}

Introduction. We will consider a real valued convex function $f$ defined on a nonempty closed convex subset $C$ of a real Banach space $E$. If $C$ has nonempty interior int $C$ and $f$ is continuous, then it is possible for $f$ to have points of differentiability in int $C$. There has been a great deal of work on such questions, mostly formulated in the following way: Determine which Banach spaces are Asplund spaces, or are weak Asplund spaces, where the former are defined to be those for which every convex continuous function on an open convex set is Fréchet differentiable at the points of a dense $G_{\delta}$ subset of its domain, and the latter are defined analogously, using Gateaux differentiability. For example, the first such theorem was proved in 1933 by S. Mazur, who showed that separable Banach spaces are (in present-day language) weak Asplund spaces. More generally, Asplund [1] showed that weakly compactly generated spaces have the same property, and that reflexive spaces are Asplund spaces. In an interesting new approach, Verona [12] recently generalized Mazur's theorem to sets $C$ which may have empty interior. She did so by substituting the set $N(C)$ of nonsupport points of $C$ for the interior of $C$. We recall some definitions.

DEFInition. A point $x \in C$ is called a support point of $C$ provided there exists a nonzero $x^{*} \in E^{*}$ such that

$$
\left\langle x^{*}, x\right\rangle=\sup \left\{\left\langle x^{*}, y\right\rangle: y \in C\right\} \equiv M\left(x^{*}, C\right) .
$$

The set of all points in $C$ which are not support points is denoted by $N(C)$.

DEFINITION. If $x \in C$, we denote by $C_{x}$ the cone generated by $C$ from $x$, that is, $y \in C_{x}$ provided there exists $t>0$ such that $x+t y \in C$. Equivalently, $C_{x}=\bigcup\{\lambda(C-x): \lambda>0\}$. (This latter description makes it obvious that $C_{x}$ is a convex cone with vertex at the origin.)

The following proposition contains some elementary but important observations (which show that whenever it is nonempty, $N(C)$ behaves very much like int $C$ ). The proofs are either very easy or are to be found in the indicated references.

Received by the editors April 15, 1987.

1980 Mathematics Subject Classification (1985 Revision). Primary 46A55, 46B99, 46G05, 26A96; Secondary 52A07, 26E15.

Key words and phrases. Convex functions, convex sets, support points, Asplund spaces, weak Asplund spaces, usco maps. 
1. PROPOSITION. For any closed nonempty convex set $C$, one has

(a) $N(C)=\operatorname{int} C$ if the latter is nonempty.

(b) $N(C)$ is empty if $C$ is contained in a closed hyperplane.

(c) $N(C)$ is convex.

(d) If $x \in N(C)$ and $y \in C$, then $[x, y) \subset N(C)$; thus, if $N(C)$ is nonempty, then it is dense in $C$.

(e) $N(C)$ is a $G_{\delta}$ subset of $C$ [9] (possibly empty), hence is a Baire space.

(f) $x \in N(C)$ if and only if $C_{x}$ is dense in $E$.

(g) If $E$ is separable and $C$ is not contained in a closed hyperplane, then $N(C)$ is nonempty [5, Example 3, p. 460].

(h) If $C$ is the positive cone in $l^{2}(\Gamma), \Gamma$ uncountable, then $N(C)$ is empty.

Recall that a continuous convex function on an open convex set is necessarily locally Lipschitzian. As we will see by the example below, even in Hilbert space, $N(C)$ does not mimic int $C$ in this latter sense: There exist continuous convex functions which are not locally Lipschitzian on $N(C)$.

DEFINITION. The subdifferential $\partial f(x)$ of the convex function $f$ at the point $x \in C$ is defined to be the set of all $x^{*} \in E^{*}$ satisfying

$$
\left\langle x^{*}, y-x\right\rangle \leq f(y)-f(x) \text { for all } y \in C .
$$

An easily verified equivalent formulation is that $x^{*} \in \partial f(x)$ if and only if

$$
\left\langle x^{*}, v\right\rangle \leq \lim _{t \rightarrow 0^{+}} \frac{f(x+t v)-f(x)}{t}
$$

whenever $v \in C_{x}$. The subdifferential is always weak* compact and convex, but it can be empty.

2. THEOREM (VERONA [12]). If $f$ is convex on $C$ and locally Lipschitzian on $N(C)$, then the subdifferential of $f$ is nonempty at each point of $N(C)$.

The idea of Verona's proof is to show that if $x \in N(C)$, then for each direction $y \in C_{x}$, the right-hand directional derivative of $f$ at $x$ exists and (using the local Lipschitzian property) defines a uniformly continuous sublinear functional $p$ on the dense cone $C_{x}$. There is a unique continuous extension of $p$ to all of $E$, and its (nonempty) subdifferential at 0 is also the subdifferential of $f$ at $x$.

It is worth observing that the convex function $f$ on $C=[0,1]$ which equals 0 on $N(C) \equiv(0,1)$ and equals 1 at the endpoints shows that the locally Lipschitzian hypothesis does not imply continuity of $f$ on all of $C$. More importantly, the following example shows that the local Lipschitzian hypothesis is essential to Verona's theorem. (In view of the latter, it also shows that a continuous convex function need not be locally Lipschitzian.)

EXAMPLE. Let $C=\left\{x \in l^{2}:\left|x_{n}\right| \leq 2^{-n}, n=1,2,3, \ldots\right\}$ and define $f$ on $C$ by $f(x)=\sum-\left(2^{-n}+x_{n}\right)^{1 / 2}$. Then $f$ is convex and continuous, but its subdifferential $\partial f(x)$ is empty for each $x \in N(C)$.

PROOF. Each summand in the definition of $f$ is continuous and convex and is dominated in absolute value by $2^{-(n-1) / 2}$, hence the series is uniformly convergent; this shows that $f$ is continuous and convex. It is easily verified that $N(C)=\{x \in$ $\left.l^{2}:\left|x_{n}\right|<2^{-n}, n=1,2,3, \ldots\right\}$. Suppose that $x \in N(C)$ and that $\partial f(x)$ were nonempty. Then there would exist an element $u \in l^{2}$ such that for each $y \in C_{x}$, the 
quantity $\langle u, y\rangle$ would be dominated by the right-hand derivative of $f$ at $x$ in the direction $y$; in particular, this would be true for $y=e_{n}$, the $n$th basis vector for $l^{2}$. (The latter is easily seen to be in $C_{x}$.) But a straightforward computation shows that the right-hand derivative of $f$ at $x$ in the direction $e_{n}$ is $-\frac{1}{2}\left(2^{-n}+x_{n}\right)^{-1 / 2}$, which implies that $u_{n} \rightarrow-\infty$ as $n \rightarrow \infty$.

3. Proposition. If $f$ is convex on $C$ and locally Lipschitzian on $N(C)$, then the set valued subdifferential map $x \rightarrow \partial f(x)$ is locally bounded and norm-to-weak ${ }^{*}$ upper semicontinuous on $N(C)$.

Recall that the set-valued map $\partial f$ is said to be locally bounded if for each point $x$ there exist a neighborhood $U$ of $x$ and a positive constant $M$ such that $\left\|x^{*}\right\| \leq M$ whenever $y \in U$ and $x^{*} \in \partial f(y)$. One can take $M$ to be the local Lipschitz constant for $f$ in a neighborhood of $x$. The proof of this proposition is well known for continuous convex functions on open convex sets, and no change is required here.

Definition. We say that $f$ is Gateaux differentiable at $x \in N(C)$ provided $\partial f(x)$ is single-valued; equivalently, provided there exists a unique $x^{*} \in E^{*}$ such that

$$
\left\langle x^{*}, y-x\right\rangle \leq f(y)-f(x) \text { for all } y \in C .
$$

We say that $f$ is Fréchet differentiable at $x \in N(C)$ provided there is a unique such $x^{*}$ (that is, it is Gateaux differentiable at $x$ ) which satisfies the following: For all $\varepsilon>0$ there exists $\delta>0$ such that $y \in N(C)$ and $\|x-y\|<\delta$ imply that

$$
0 \leq f(y)-f(x)-\left\langle x^{*}, y-x\right\rangle \leq \varepsilon\|y-x\| .
$$

These definitions coincide with the usual ones when $C$ has nonempty interior (and hence $N(C)=\operatorname{int} C$ ).

The proof of the following proposition is standard for open convex sets, and applies to this situation essentially without change. (See, for instance, [4, p. 134].) For completeness, we sketch a proof of the direction which we will need.

4. PROPOSITION. If $f$ is convex on $C$ and locally Lipschitzian on $N(C)$, then it is Gateaux [Fréchet] differentiable at a point $x \in N(C)$ if (and only if) there is a selection $\varphi$ for the subdifferential map which is norm-to-weak ${ }^{*}$ [norm-to-norm] continuous at $x$.

ProOF. Suppose that such a selection $\varphi$ exists. Since $\varphi(x) \in \partial f(x)$, we must have $\langle\varphi(x), y-x\rangle \leq f(y)-f(x)$ for all $y \in N(C)$. For such $y$ we also have $\varphi(y) \in \partial f(y)$, so $\langle\varphi(y), x-y\rangle \leq f(x)-f(y)$. These inequalities combine to show that for all $y \in N(C)$,

$$
0 \leq f(y)-f(x)-\langle\varphi(x), y-x\rangle \leq\langle\varphi(y)-\varphi(x), y-x\rangle .
$$

If $\varphi$ is norm-to-weak ${ }^{*}$ continuous at $x$, one can use the local boundedness of $\varphi$, make the substitution $y=x+t v\left(v \in C_{x}, t>0\right.$ sufficiently small), divide by $t$ and take the limit as $t \rightarrow 0^{+}$to conclude that $\langle\varphi(x), v\rangle$ is the directional derivative of $f$ at $x$ in the direction $v$, for each $v$ in the (dense) set $C_{x}$. This implies that $\varphi(x)$ is the unique element of $\partial f(x)$. If $\varphi$ is norm-to-norm continuous at $x$, then the fact the last term in $(*)$ is dominated by $\|\varphi(y)-\varphi(x)\| \cdot\|y-x\|$ shows that $f$ is Fréchet differentiable at $x$. 
DEFINITION. A set-valued map $F$ from a Hausdorff space $X$ into the nonempty compact subsets of another Hausdorff space $Y$ is said to be usco (uppersemicontinuous and compact valued) provided $\{x \in X: F(x) \subset U\}$ is open in $X$ for every open subset $U$ of $Y$. The space $Y$ is said to be of type $\mathbf{S}$ if for every usco map $F$ from a Baire space $X$ into $2^{Y}$ (the space of all subsets of $Y$ ) there exist a selection $\sigma$ for $F$ and a dense $G_{\delta}$ subset $D$ of $X$ such that $\sigma$ is continuous at each point of $D$. Finally, a Banach space $E$ is said to be of class $(S)$ provided $\left(E^{*}\right.$, weak $\left.{ }^{*}\right)$ is of type $\mathbf{S}$.

The foregoing class of spaces was introduced and investigated by Stegall $[\mathbf{1 0}$, 11], who showed that $E$ is of class $(S)$ if and only if the unit ball of $E^{*}$ is of type $\mathbf{S}$ in the weak ${ }^{*}$ topology. He also showed that every space of class $(S)$ is a weak Asplund space and that the class possesses many desirable permanence properties. It is easy to see that metric spaces are of type $\mathbf{S}$. More generally, one can prove the following proposition.

5. Proposition. Let $(Y, \tau)$ be a Hausdorff space and let $F$ be a usco map from a Baire space $X$ into $2^{Y}$. Suppose that there is a metric d on $Y$ with the following property: For each nonempty open subset $U$ of $X$, there exists a nonempty open subset $V$ of $U$ such that each nonempty subset of $F(V)$ contains nonempty relatively open subsets of arbitrarily small d-diameter. [Here, $F(V)=\bigcup\{F(x): x \in V\}$.] Then there exist a selection $\sigma$ for $F$ and a dense $G_{\delta}$ subset $D$ of $X$ such that $\sigma$ is both $\tau$ - and $d$-continuous at each point of $D$.

Proof (SKetch). Let $G_{F}=\{(x, y) \in X \times Y: y \in F(x)\}$ be the graph of $F$. From standard facts about usco maps (see, for instance, [2]), we may assume that $F$ is a minimal usco map, that is, if $C$ is a proper closed subset of $G_{F}$, then $p[C] \neq X$, where $p: X \times Y \rightarrow X$ is the projection. For a given $\varepsilon>0$, let

$$
O_{\varepsilon}=\bigcup\{G: G \text { is an open subset of } X \text { and } d \text {-diam } F(G) \leq \varepsilon\} \text {. }
$$

Clearly, $O_{\varepsilon}$ is open in $X$; we will show that it is dense. Let $U$ be a nonempty open subset of $X$. By hypothesis, there is a nonempty open subset $V$ of $U$ and a $\tau$-open subset $W$ of $Y$ such that $F(V) \cap W \neq \varnothing$ and $d$-diam $(F(V) \cap W) \leq \varepsilon$. Since $G_{F} \cap(V \times W) \neq \varnothing$ and $F$ is minimal, $p\left[G_{F} \backslash(V \times W)\right] \neq X$. Choose $x_{0} \in X \backslash p\left[G_{F} \backslash(V \times W)\right]$. Then $p^{-1}\left(x_{0}\right) \cap G_{F} \subset V \times W$, that is, $x_{0} \in V$ and $F\left(x_{0}\right) \subset W$. If $G=\{x \in X: F(x) \subset W\} \cap V$, then $G$ is an open neighborhood of $x_{0}$ such that $d$-diam $F(G) \leq d$-diam $(F(V) \cap W) \leq \varepsilon$. It follows that $G \subset O_{\varepsilon}$ and therefore $\varnothing \neq V \cap O_{\varepsilon} \subset U \cap O_{\varepsilon}$. This proves that $O_{\varepsilon}$ is dense in $X$. Now let $D=\bigcap\left\{O_{1 / n}: n \in N\right\}$. Since $X$ is a Baire space, $D$ is a dense $G_{\delta}$ subset of $X$. Clearly $F(x)$ is a singleton for each $x \in D$. If $\sigma$ is any selection for $F$, then $\sigma$ is $\tau$-continuous at each point of $D$ since $F$ is upper semicontinuous. From the definition of $D$, it is also clear that $\sigma$ is $d$-continuous at each point of $D$.

If $K$ is a nonempty weakly compact subset of a Banach space, then the identity map from $(K$, weak) to $(K$, norm $)$ is continuous at some point of $K$ (cf. [7]). Thus, there are relatively weakly open subsets of $K$ of arbitrarily small diameter. In view of this fact and Proposition 5, we see that ( $K$, weak) is of type $\mathbf{S}$. A compact space is said to be Eberlein compact (EC) if it is homeomorphic to a weakly compact subset of a Banach space. Hence each EC space is of type $\mathbf{S}$. If $E$ is a weakly compactly generated (WCG) Banach space, then the unit ball of the dual space 
$E^{*}$ is EC in the weak* topology (see, for instance, [3]). Hence, any WCG space is of class $(S)$. In particular, any separable space, reflexive space or $L^{1}$ space (over a $\sigma$-finite measure space) is of class $(S)$.

6. THEOREM. If the Banach space $E$ is of class $(S)$, in particular, if $E$ is weakly compactly generated, then for every closed convex subset $C$ of $E$ such that $N(C)$ is nonempty and every convex function $f$ on $C$ which is locally Lipschitzian on $N(C)$, the set of points $G$ where $f$ is Gateaux differentiable is a dense $G_{\delta}$ subset of $C$.

PROOF. The proof is straightforward; since $N(C)$ is a Baire space and since the subdifferential map is usco, we need only apply the definitions and Proposition 4 to obtain the differentiability conclusion.

PROBLEM. Is the conclusion to the foregoing theorem valid in any weak Asplund space?

7. THEOREM. Suppose that $E$ is an Asplund space and that $C$ is a closed convex subset of $E$ such that $N(C)$ is nonempty. If $f: C \rightarrow R$ is convex on $C$ and locally Lipschitzian on $N(C)$, then there exists a dense $G_{\delta}$ subset $D$ of $N(C)$ such that the subdifferential map $x \rightarrow \partial f(x)$ (defined at each point of $N(C)$ ) is norm-norm continuous at each point of $D$; equivalently, $f$ is Fréchet differentiable at each point of $D$.

PROOF. The proof is similar to the one for Theorem 6. The subdifferential map $x \rightarrow \partial f(x)$ is usco from the Baire space $N(C)$ to the nonempty weak* compact subsets of $E^{*}$. The hypotheses of Proposition 5 are satisfied with $(Y, \tau)=\left(E^{*}\right.$, weak $\left.^{*}\right)$, $X=N(C), d=$ the norm metric and $F(x)=\partial f(x)$. In fact, let $U$ be a nonempty open subset of $N(C)$. Since $\partial f$ is locally bounded (Proposition 3 ), there is a nonempty open subset $V$ of $U$ such that $\partial f(V)$ is bounded. By Lemma 3 of [8], each nonempty subset of $\partial f(V)$ contains relatively weak* ${ }^{*}$-open nonempty subsets of arbitrarily small norm diameter. Hence by Proposition 5 , there are a selection $\sigma$ for $\partial f$ and a dense $G_{\delta}$ subset $D$ of $N(C)$ such that $\sigma$ is norm-to-norm continuous at each point of $D$. By Proposition 4, this means that $f$ is Fréchet differentiable at each point of $D$.

In the case when int $C$ is nonempty and $f$ is merely assumed to be continuous and convex, Theorem 6 has been proved and Theorem 7 announced by Stegall $[\mathbf{1 0}$, 11]. An alternative proof of Theorem 7 can be obtained by modifying Kenderov's proof [6] that monotone operators on Asplund spaces have a dense $G_{\delta}$ set of points of norm-to-norm upper semicontinuity.

ACKNOWLEDGEMENT. The author wishes to thank Professors I. Namioka and R. R. Phelps for very helpful conversations on the subject matter of this note.

\section{REFERENCES}

1. Edgar Asplund, Fréchet differentiability of convex functions, Acta Math. 121 (1968), 31-47.

2. J. P. R. Christensen, Theorems of Namioka and R. E. Johnson type for upper semicontinuous and compact-valued set-valued mappings, Proc. Amer. Math. Soc. 86 (1982), 649-655.

3. Joseph Diestel, Geometry of Banach spaces -- selected topics, Lecture Notes in Math., vol. 485, Springer-Verlag, 1975.

4. John Giles, Convex analysis with application to differentiation of convex functions, Research Notes in Math., no. 58, Pitman, Boston, Mass., 1982. 
5. R. B. Holmes, Geometric functional analysis and its applications, Springer-Verlag, 1975.

6. P. S. Kenderov, Monotone operators in Asplund spaces, C. R. Acad. Bulgare Sci. 30 (1977), 963-964.

7. I. Namioka, Separate continuity and joint continuity, Pacific J. Math. 51 (1974), 515-531.

8. Isaac Namioka and R. R. Phelps, Banach spaces which are Asplund spaces, Duke Math. J. 42 (1975), 735-749.

9. R. R. Phelps, Some topological properties of support points of convex sets, Israel J. Math. 13 (1972), 327-336.

10. C. Stegall, A class of topological spaces and differentiation of functions on Banach spaces, Proc. Conf. on Vector Measures and Integral Representations of Operators, Vorlesungen aus dem Fachbereich. Math., Heft 10 (W. Ruess, ed.), Univ. Essen, 1983.

11. _ More Gateaux differentiability spaces, Proc. Conf. Banach Spaces, Univ. Missouri, 1984 (N. Kalton and E. Saab, eds.), Lecture Notes in Math., vol. 1166, Springer-Verlag, 1985, pp. 158-168.

12. M. E. Verona, More on the differentiability of convex functions, Proc. Amer. Math. Soc. 103 (1988), 137-140.

Department of Mathematics, University of Washington, Seattle, WashingTON, 98195 